\title{
ZEITSCHRIFT FÜR KRISTALLOGRAPHIE NEW CRYSTAL STRUCTURES
}

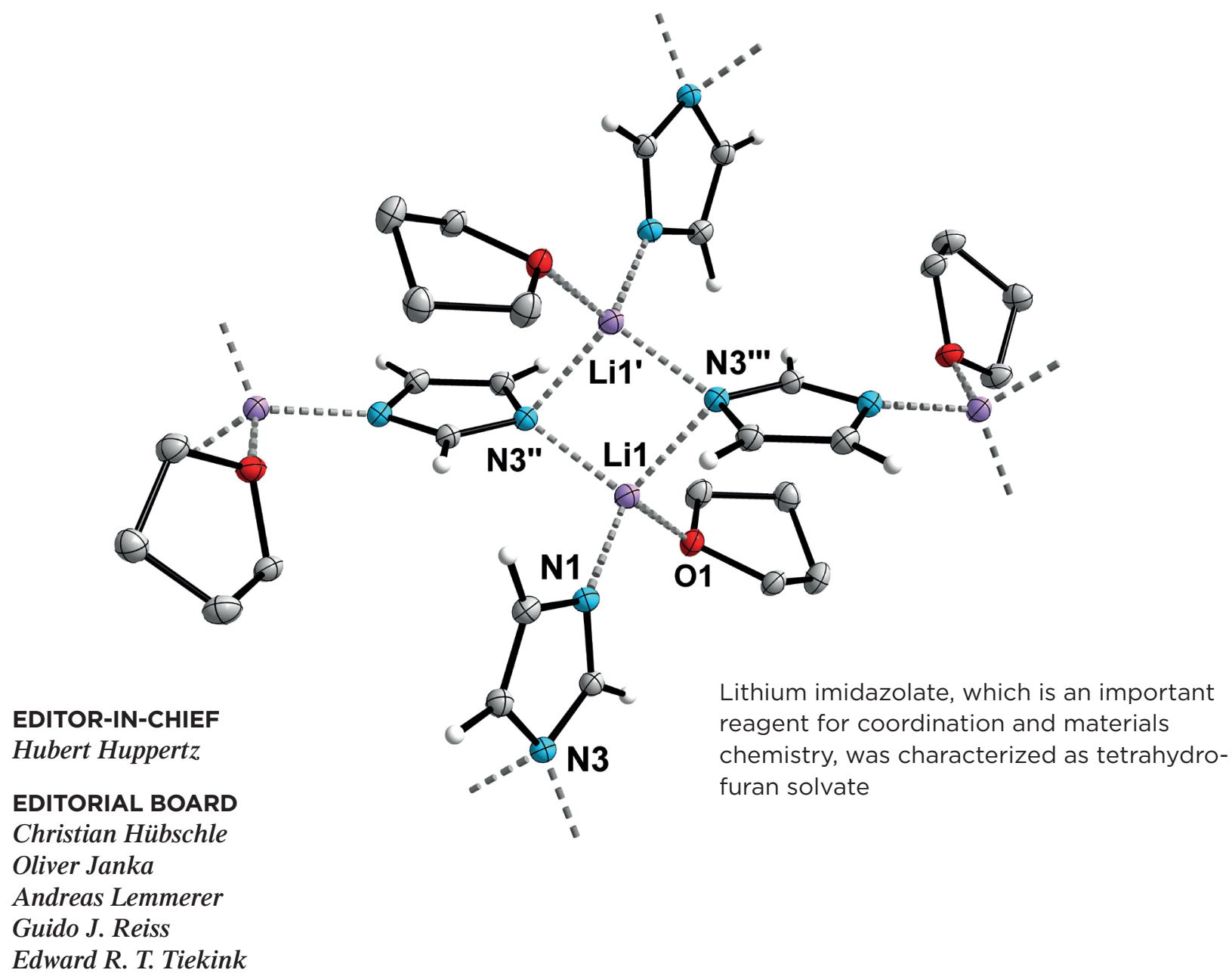




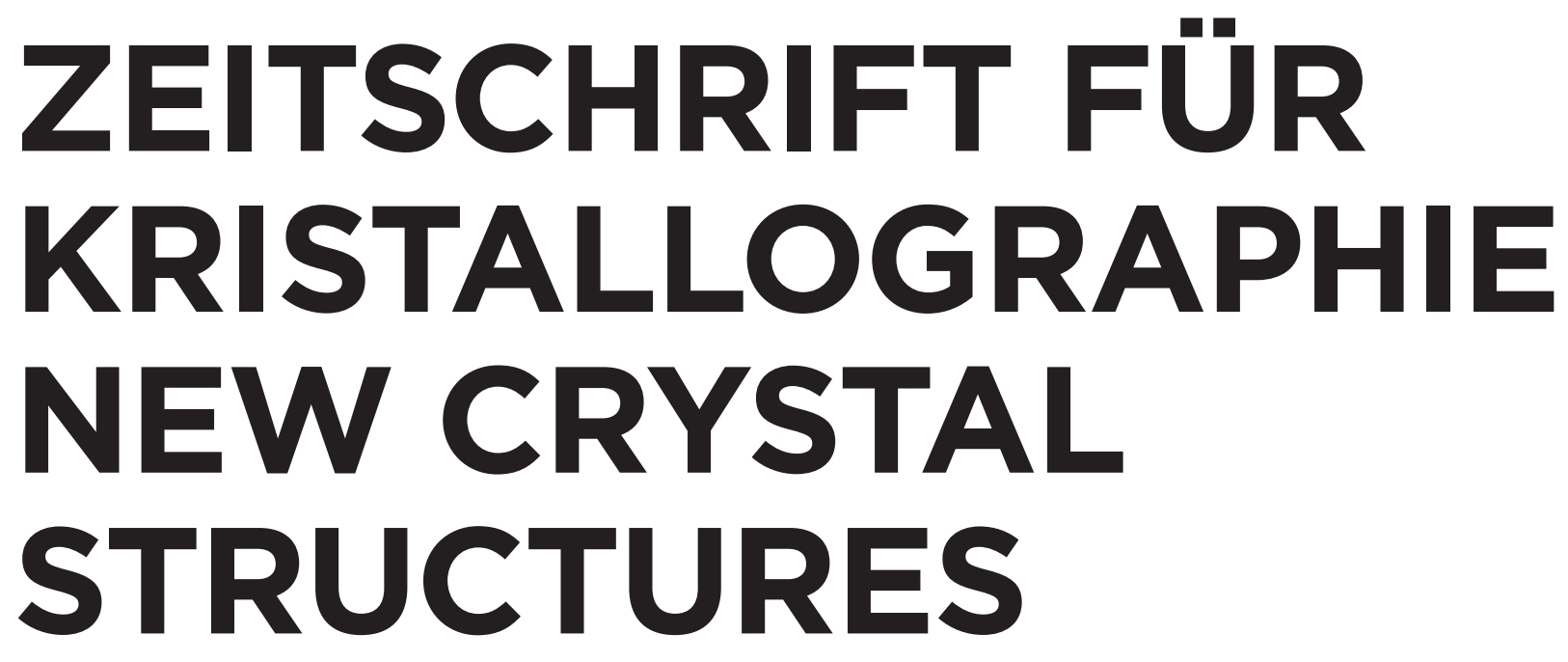

EDITOR-IN-CHIEF

Hubert Huppertz

EDITORIAL BOARD

Christian Hübschle

Oliver Janka

Andreas Lemmerer

Guido J. Reiss

Edward R. T. Tiekink

DE GRUYTER 
Zeitschrift für Kristallographie - New Crystal Structures was founded in 1997 as spin off of Zeitschrift für Kristallographie to meet the high demand to publish results of structure determination. All publications and their submitted CIF-data are online freely available (Open Access). Zeitschrift für Kristallographie - New Crystal Structures offers a place for researchers to present

- results of determinations of hitherto unknown crystal structures which do not justify detailed discussion of determination procedure, crystal structure, and/or structure-property relations (routine determinations and structures),

- refinement of previously published crystal structures which do not require a new description or discussion.

Further details of the structure determination can be accessed at the relevant database. Data with CSD numbers can be obtained from the Fachinformationszentrum Karlsruhe, Gesellschaft für wissenschaftlichtechnische Information mbH, 76344 Eggenstein-Leopoldshafen, Germany (e-mail: crysdata@ fiz-karlsruhe.de). Data with CCDC numbers can be obtained from the Cambridge Crystallographic Data Centre (CCDC), 12 Union Road, Cambridge CB2 1EZ, UK, (fax: +44-(0)1223-33 6033 or e-mail: deposit@chemcrys.cam.ac.uk).

ONLINE-SUBMISSION New Crystal Structures (NCS) have to be submitted online as CIF-file to: https://mc.manuscriptcentral.com/zkrist-ncs

ABSTRACTED/INDEXED IN Baidu Scholar · Cabell's Directory · Celdes · Chemical Abstracts Service (CAS): CAplus; SciFinder · CNKI Scholar (China National Knowledge Infrastructure) · CNPIEC · EBSCO (relevant databases) · EBSCO Discovery Service · Elsevier: Reaxys; SCOPUS · Genamics JournalSeek · GeoRef · Google Scholar · Inspec · J-Gate · JournalTOCs · KESLI-NDSL (Korean National Discovery for Science Leaders) · Naviga (Softweco) $\cdot$ Paperbase $\cdot$ Pirabase $\cdot$ Polymer Library $\cdot$ Primo Central (ExLibris) $\cdot$ ReadCube $\cdot$ ResearchGate $\cdot$ SCImago (SJR) $\cdot$ Summon (Serials Solutions/ProQuest) · TDNet · TEMA Technik und Management · Thomson Reuters: Current Contents/Physical, Chemical and Earth Sciences; Journal Citation Reports/Science Edition; Science Citation Index Expanded · WanFang Data · WorldCat (OCLC).

ISSN $1433-7266 \cdot$ e-ISSN 2197-4578

All information regarding notes for contributors, subscriptions, Open access, back volumes and orders is available online at www.degruyter.com/ncrs

RESPONSIBLE EDITOR Univ.-Prof. Dr. Hubert Huppertz, University of Innsbruck, Institute of General, Inorganic and Theoretical Chemistry, Innrain 80-82, 6020 Innsbruck, Austria, e-mail: Hubert.Huppertz@uibk.ac.at

PUBLISHER Walter de Gruyter GmbH, Berlin/Boston, Genthiner Straße 13, 10785 Berlin, Germany

JOURNAL MANAGER Holger Kleessen, De Gruyter, Genthiner Straße 13, 10785 Berlin, Germany, Tel.: +49 (0)30 26005 - 348, Fax: +49 (0)30 26005 - 184, e-mail: ZKristallogr-NCS@ degruyter.com

RESPONSIBLE FOR ADVERTISEMENTS Markus Kügel, De Gruyter, Rosenheimer Str. 143, 81671 München, Germany, Tel.: +498976 902-424, e-mail: anzeigen@ degruyter.com

(C) 2021 Walter de Gruyter GmbH, Berlin/Boston, Germany

TYPESETTING TNQ Technologies, Chennai, India

PRINTING Franz X. Stückle Druck und Verlag e.K., Ettenheim

COVER ILLUSTRATION Ludwig Zapf and Maik Finze: The crystal structure of poly[( $\mu 3$-imidazolato- $\left.\kappa^{3} N: N: N^{\prime}\right)\left(\right.$ tetrahydrofuran- $\left.\kappa^{1} O\right)$ lithium(I)], $\mathrm{C}_{7} \mathrm{H}_{11} \mathrm{LiN}_{2} \mathrm{O}$; in this issue pp. 1007-1009. DOI: 10.1515/ncrs-2021-0192.

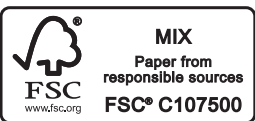




\section{Contents}

Benlian LV

Crystal structure of [aqua-(4-iodopyridine-2,6dicarboxylato-k $\left.{ }^{3} O, N, O^{\prime}\right)-\left(1,10\right.$-phenanothroline- $\left.\kappa^{2} N, N^{\prime}\right)$ copper(II)] dihydrate, $\mathrm{C}_{19} \mathrm{H}_{16} \mathrm{O}_{7} \mathrm{~N}_{3} \mathrm{Cul}-891$

Bingchuan Yang, Xueyan Lv and Rutao Liu The crystal structure of tetrakis(1-isopropyl-1Himidazolium) octamolybdate, $\mathrm{C}_{24} \mathrm{H}_{44} \mathrm{Mo}_{8} \mathrm{~N}_{8} \mathrm{O}_{26}-895$

Wei-Dong Yin, Qian-Long Liu, Ya-Jing Zhao, Xiao-Ru Gong and Gui-Lian Li

Crystal structure of catena-poly[bis $\left(\mu_{2}-3,5\right.$-bis

(1-imidazolyl)pyridine- $\left.{ }^{2} N: N^{\prime}\right)-\left(\mu_{2}-3\right.$-nitrophthalato-

$\left.\mathrm{k}^{3} O, O^{\prime}: O^{\prime \prime}\right)$ cadmium(II)] dihydrate,

$\mathrm{C}_{30} \mathrm{H}_{25} \mathrm{~N}_{11} \mathrm{O}_{8} \mathrm{Cd}-899$

Zhang Yong-Hua, Meng-Yao Wen and Ke Yan

The crystal structure of diaqua-bis(2-(3-(1H-pyrazol-4-yl)$1 H$-1,2,4-triazol-5-yl)pyridine- $\left.\kappa^{2} N: N^{\prime}\right)$-bis(3,5-

dicarboxybenzoato-k ${ }^{1} O$ )cobalt(II),

$\mathrm{C}_{38} \mathrm{H}_{30} \mathrm{CoN}_{12} \mathrm{O}_{14}-903$

Wang Yu-Fang and Shu-Qi Zhang

Crystal structure of the nickel(II) complex aqua-(2,6-

di(pyrazin-2-yl)-4, $4^{\prime}$-bipyridine- $\left.\kappa^{3} N, N^{\prime}, N^{\prime}\right)$-(phthalato-

$\mathrm{K}^{2} 0,0$ ) nickel(II) tetrahydrate, $\mathrm{C}_{26} \mathrm{H}_{26} \mathrm{~N}_{6} \mathrm{O}_{9} \mathrm{Ni}-907$

Yi-Xia Gong, Yi-Ding Geng, Shu-Jing Zhou and

Gui-Yan Wang

The crystal structure of 1-[5-(2-fluorophenyl)-1-(pyridine3-sulfonyl)-1 $\mathrm{H}$-pyrrol-3-yl]- $\mathrm{N}$-methylmethanaminium 3carboxyprop-2-enoate, $\mathrm{C}_{21} \mathrm{H}_{20} \mathrm{FN}_{3} \mathrm{O}_{6} \mathrm{~S}-911$

Jie Cheng and Jue Chen

The crystal structure of 1,2-bis(4-pyridyl)ethane - 4,4dihydroxydiphenylmethane (1/1), $\mathrm{C}_{25} \mathrm{H}_{21} \mathrm{~N}_{2} \mathrm{O}_{2}-915$

Ze-Sen Jin, Xiao-jing Liu, E. Liu, Tongling Liang and Fangfang Jian

Crystal structure of bis(2-((E)-5-chloro-2-

hydroxybenzylidene)hydrazineyl)methaniminium

trifluoroacetate dihydrate, $\mathrm{C}_{34} \mathrm{H}_{36} \mathrm{Cl}_{4} \mathrm{~N}_{10} \mathrm{O}_{12}-919$

Chun-Yan Zhang, Xu-Liang Nie, Guo-Ping Huang,

Yuan-Zhen Xiong and Jian-Ping Huang

Crystal structure of 1-cyclopropyl-7-ethoxy-6,8-difluoro-

4-oxo-1,4-dihydro-quinoline-3-carboxylic acid,

$\mathrm{C}_{15} \mathrm{H}_{13} \mathrm{~F}_{2} \mathrm{NO}_{4}-923$
Qiang Ma and Zong-Cheng Miao

Crystal structure of methyl 3-(1H-naphtho[1,8-de][1,3,2]

diazaborinin-2(3H)-yl)benzoate, $\mathrm{C}_{18} \mathrm{H}_{15} \mathrm{BN}_{2} \mathrm{O}_{2}-927$

Jianlian Liu, Hong Hao and Chaojun Du

Crystal structure of (E)- $N^{\prime}$-(2-chloro-6-

hydroxybenzylidene)-2-hydroxybenzohydrazide,

$\mathrm{C}_{14} \mathrm{H}_{11} \mathrm{ClN}_{2} \mathrm{O}_{3}-929$

Chen Aiqing and Zhang Lixue

Crystal structure of Al-rich fluorophlogopite,

$\mathrm{K}_{1.0}\left(\mathrm{Mg}_{2.8} \mathrm{Al}_{0.2}\right)\left(\mathrm{Si}_{2.8} \mathrm{Al}_{1.2}\right) \mathrm{O}_{10} \mathrm{~F}_{2}-931$

Xingyu $X u$ and Ping Qiu

The crystal structure of 4,5-diiodo-1,3-dimesityl-1H-1,2,3triazol-3-ium hexafluoridoantimonate $(\mathrm{V})$,

$\mathrm{C}_{20} \mathrm{H}_{22} \mathrm{~F}_{6} \mathrm{I}_{2} \mathrm{~N}_{3} \mathrm{Sb}-935$

Yuxin Ma, Munan Hao, Ruijin Sun, Shifeng Jin and

Changchun Zhao

Crystal structure of tris(3-iodopyridin-1-ium) catena-poly [(hexachlorido- $\left.\mathrm{\kappa}^{1} \mathrm{Cl}\right)$ - $\left(\mu_{2}\right.$-trichlorido- $\left.\mathrm{\kappa}^{2} \mathrm{Cl}: \mathrm{Cl}\right)$

diantimony(III)], $\mathrm{C}_{15} \mathrm{H}_{15} \mathrm{Cl}_{9} \mathrm{I}_{3} \mathrm{~N}_{3} \mathrm{Sb}_{2}-937$

Wenqi Song and Zongcheng Miao

Crystal structure of methyl 2-(1H-naphtho[1,8-de]

[1.3.2] diazaborinin-2(3H-yl)benzoate

$\mathrm{C}_{18} \mathrm{H}_{15} \mathrm{BN}_{2} \mathrm{O}_{2}-941$

Miyuki Kobayashi, Kikuko lida, Hiroaki litsuka, Kun Li, Noriyuki Yonezawa and Akiko Okamoto

The crystal structure of 1,8-bis(4-methoxybenzoyl)

naphthalene-2,7-diyl dibenzoate, $\mathrm{C}_{40} \mathrm{H}_{28} \mathrm{O}_{8}-945$

Weifeng He, Yingfan Liu, Saisai Sun, Guangqian Ji and Xiaochuan Li

Crystal structure of 2-bromo-1,3,6,8-

tetramethylBOPHY (BOPHY = bis(difluoroboron)-1,2-

bis((1H-pyrrol-2-yl)methylene)hydrazine),

$\mathrm{C}_{14} \mathrm{H}_{15} \mathrm{~B}_{2} \mathrm{BrF}_{4} \mathrm{~N}_{4}-949$

Hui-Zhen Wu, Li-Jing Min, Liang Han, Xing-Hai Liu and Na-Bo Sun

The crystal structure of (E)-3-chloro-2-(2-(2-

fluorobenzylidene)hydrazinyl)pyridine,

$\mathrm{C}_{12} \mathrm{H}_{9} \mathrm{ClFN}_{3}-953$ 
Benlian Lv

Crystal structure of bis $\left(\mu_{2}\right.$ - 4-iodopyridine-2,6dicarboxylato- $\left.{ }^{3} \mathrm{O}: \mathrm{N}: \mathrm{O}^{\prime}\right)$-bis(4-iodopyridine-2,6dicarboxylato- $\left.{ }^{3} \mathrm{O}: \mathrm{N}: \mathrm{O}^{\prime}\right)$-bis $\left(\mu_{2}-1\right.$-(4-pyridyl)piperazine$\left.\kappa^{2} N: N^{\prime}\right)$-hexa-aqua-tetra-copper(II),

$\mathrm{C}_{46} \mathrm{H}_{46} \mathrm{Cu}_{4} \mathrm{I}_{4} \mathrm{~N}_{10} \mathrm{O}_{22}-957$

Benlian Lv and Yunli Ren

Crystal structure of poly[diaqua- $\left(\mu_{2}-2,5-\right.$

dihydroxyterephthalato- $\left.\mathrm{\kappa}^{2} \mathrm{O}: \mathrm{O}^{\prime}\right)\left(\mu_{2}\right.$-bis(4-pyridylformyl) piperazine- $\left.\mathrm{K}^{2} \mathrm{~N}: \mathrm{N}^{\prime}\right)$ cadmium(II)] dihydrate,

$\mathrm{C}_{24} \mathrm{H}_{28} \mathrm{CdN}_{4} \mathrm{O}_{12}-961$

Yu-Mei Guo, Bo Tang, Hong-Guang Ge, Pei Huang and Jiu-Fu Lu

Crystal structure of poly[aqua-( $\mu_{2}-1,3-\mathrm{di}(1 \mathrm{H}$-imidazol-1-yl) propane- $\left.{ }^{2} N: N^{\prime}\right)-\left(\mu_{3}-2,3,5,6\right.$-tetrafluoroterephthalato$\kappa^{3} O: O^{\prime}: O^{\prime \prime}$ )cadmium(II)], $\mathrm{C}_{17} \mathrm{H}_{14} \mathrm{~N}_{4} \mathrm{O}_{5} \mathrm{~F}_{4} \mathrm{Cd}-965$

Chuan-Jun Li

Crystal structure of 6-(quinolin-8-yl)benzo[a]

phenanthridin-5(6H)-one, $\mathrm{C}_{26} \mathrm{H}_{16} \mathrm{~N}_{2} \mathrm{O}-969$

Zhongyu Zhang, Mingqiong Tong and Yanling Liu The crystal structure of aqua-bis(6-chloropicolinato$\left.\mathrm{K}^{2} \mathrm{~N}, \mathrm{O}\right)$ copper(II), $\mathrm{C}_{12} \mathrm{H}_{8} \mathrm{Cl}_{2} \mathrm{~N}_{2} \mathrm{O}_{5} \mathrm{Cu}-971$

Han-Lin Zhao, Jin-Ke Jiang, Hui Jiang, Xian-Wu Dong and Xue Li

Crystal structure of catena-poly[diaqua-bis $\left(\mu_{2}-4,4^{\prime}\right.$ bipyridyl- $\left.{ }^{2} N: N^{\prime}\right)$ disilver(I)] 4-oxidopyridine-3-sulfonate trihydrate, $\mathrm{C}_{25} \mathrm{H}_{29} \mathrm{Ag}_{2} \mathrm{~N}_{5} \mathrm{O}_{9} \mathrm{~S}-973$

Arshad Khan, Rabia Usman, Amjad Khan, Moamen S. Refat, Abeer M. Alosaimi, Safyah B. Bakare and Mohammed T. Alghamdi

The crystal structure of 4-(3-bromophenyl)pyrimidin-2amine, $\mathrm{C}_{10} \mathrm{H}_{8} \mathrm{BrN}_{3}-975$

Bei-Chen Shi, Hao-Dong Chen, Xian-Dui Zhu, Meng-Meng Zhang and Zhi-Fei Zhang

Crystal structure of 6-0xo-4-phenyl-1-propyl-1,6-

dihydropyridine-3-carbonitrile, $\mathrm{C}_{15} \mathrm{H}_{14} \mathrm{~N}_{2} \mathrm{O}-977$

Jianlian Liu, Chaojun Du and Hong Hao

Crystal structure of 4-(2,2-difluoroethyl)-2,4-dimethyl-6(trifluoromethyl)isoquinoline-1,3(2H,4H)-dione,

$\mathrm{C}_{14} \mathrm{H}_{12} \mathrm{~F}_{5} \mathrm{NO}_{2}-979$
Pengju Liu, Zongwei Wang, Libing Guo

and Junming Tang

Crystal structure of dibromido-(1-methyl- $1 \mathrm{H}$-imidazole$\kappa^{1} N$ )-(3-(3-methyl-1H-imidazol-3-ium-1-yl)propanoato$\kappa^{1} O$ )zinc(II), $\mathrm{C}_{11} \mathrm{H}_{16} \mathrm{Br}_{2} \mathrm{~N}_{4} \mathrm{O}_{2} \mathrm{Zn}-981$

Tiankun Zhao, Peng Wang, Mingyu Ji, Shanjia Li, Mingjun Yang and Xiuying $\mathrm{Pu}$

The crystal structure of 1,1'-(((2 (dimethylamino)ethyl) azanediyl)bis(methylene)) bis(naphthalen-2-olato$\kappa^{4} N, N^{\prime}, O, O^{\prime}$ )-(pyridine-2,6-dicarboxylato- $N, O, O^{\prime}$ )titanium(IV) - dichloromethane (2/1),

$\mathrm{C}_{33} \mathrm{H}_{29} \mathrm{~N}_{3} \mathrm{O}_{6} \mathrm{Ti}-985$

Guido J. Reiss and Maik Wyshusek

The layered crystal structure of bis(theophyllinium) hexachloridostannate (IV), $\mathrm{C}_{14} \mathrm{H}_{18} \mathrm{~N}_{8} \mathrm{O}_{8} \mathrm{SnCl}_{6}-989$

Yanfang Zhu, Guiyang Xu, Wenqi Song and Zongcheng Miao

The crystal structre of 3-(1-ethenyl- $\mathrm{H}$-imidazol-3-ium-3yl)propane-1-sulfonate, $\mathrm{C}_{8} \mathrm{H}_{12} \mathrm{~N}_{2} \mathrm{O}_{3} \mathrm{~S}-993$

Wu-Wu Li, Xiong-Li Liu, Xiao-Peng Li, Hao-Nan Zheng, A-Tong Weng, Ling-Ying Feng and Lu Liu

Synthesis and crystal structure of di-tert-butyl 1"-acetyl-

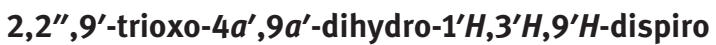
[indoline-3,2'-xanthene-4',3'"-indoline]-1,3'-

dicarboxylate, $\mathrm{C}_{39} \mathrm{H}_{38} \mathrm{~N}_{2} \mathrm{O}_{9}-995$

Fan Yang, Lin Yao and Xu-Li Zeng

The crystal structure of 4-chloro-2-(quinolin-8-yl)

isoindoline-1,3-dione, $\mathrm{C}_{17} \mathrm{H}_{9} \mathrm{ClN}_{2} \mathrm{O}_{2}-999$

Jin-Bao Liu

The crystal structure of 1-fluoro-4-( $p$-tolylethynyl) benzene, $\mathrm{C}_{15} \mathrm{H}_{11} \mathrm{~F}-1001$

Željko K. Jaćimović, Zoran D. Tomić, Gerald Giester, Eugen Libowitzky, Atifa Ajanović and Milica Kosović

The crystal structure of bis[4-bromo-2-(1H-pyrazol-3yl) phenolato- $\left.{ }^{2} \mathrm{~N}, 0\right]$ copper(II),

$\mathrm{C}_{18} \mathrm{H}_{12} \mathrm{Br}_{2} \mathrm{CuN}_{4} \mathrm{O}_{2}-1003$

Ludwig Zapf and Maik Finze The crystal structure of poly[ $\left(\mu_{3}\right.$-imidazolato- $\left.\kappa^{3} N: N: N^{\prime}\right)-$ (tetrahydrofuran- $\kappa^{1} O$ ) lithium(I)], $\mathrm{C}_{7} \mathrm{H}_{11} \mathrm{LiN}_{2} \mathrm{O}-1007$ 
Yi Zhou, Min Zhang and Ying Xiong

Crystal structure of $N^{\prime}, N^{\prime \prime \prime}-\left(\left(1 E, 1^{\prime} E\right)\right.$-(propane-2,2diylbis(1H-pyrrole-5,2diyl))bis(methaneylylidene)) di(nicotinohydrazide) pentahydrate, $\mathrm{C}_{25} \mathrm{H}_{24} \mathrm{~N}_{8} \mathrm{O}_{2}$. $5 \mathrm{H}_{2} \mathrm{O}-1011$

Kun Yuan, Wan-Ming Xiong, Xu-Liang Nie, Da-Yong Peng and Jing Chen

Crystal structure of 3-(2-ethoxy-2-oxoethyl)-1-ethyl-1Himidazol-3-ium hexafluoridophos-phate(V),

$\mathrm{C}_{9} \mathrm{H}_{15} \mathrm{~F}_{6} \mathrm{~N}_{2} \mathrm{O}_{2} \mathrm{P}-1015$

Zhongyu Zhang, Xiaoxia Men, Bing Han and Chunyan Sun Crystal structure of (1,10-phenanthroline- $\left.{ }^{2} N, N^{\prime}\right)$-bis(3thiophenecarboxylato- $\left.\mathrm{K}^{2} \mathrm{O}, \mathrm{O}^{\prime}\right)$ copper(II),

$\mathrm{C}_{22} \mathrm{H}_{14} \mathrm{~N}_{2} \mathrm{O}_{4} \mathrm{~S}_{2} \mathrm{Cu}-1019$

Guang Huang

The crystal structure of 2-amino-3-carboxypyridin-1-ium iodide hemihydrate, $\mathrm{C}_{6} \mathrm{H}_{8} \mathrm{IN}_{2} \mathrm{O}_{2.5}-1021$

Lei Wang, Qing-Guo Meng and Gui-Ge Hou Crystal structure of (E)-7-methoxy-2-((6-methoxypyridin2-yl)methylene)-tetralone, $\mathrm{C}_{18} \mathrm{H}_{17} \mathrm{NO}_{3}-1027$

Lehlohonolo Moherane, Orbett T. Alexander, Hendrik G. Visser and Amanda-Lee E. Manicum The crystal structure of [ $\mu$-hydroxido-bis[(5,5'-dimethyl2,2'-bipyridine- ${ }^{2} N, N^{\prime}$ )-tricarbonylrhenium(I)] bromide hemihydrate, $\mathrm{C}_{30} \mathrm{H}_{26} \mathrm{~N}_{4} \mathrm{O}_{9} \mathrm{Re}_{2} \mathrm{Br}-1031$

Zhi-Wei Zhai and Shuang-Hua Yang The crystal structure of 2,5-bis(3,5-dimethylphenyl) thiazolo[5,4-d] thiazole, $\mathrm{C}_{20} \mathrm{H}_{18} \mathrm{~N}_{2} \mathrm{~S}_{2}-1035$

Zeliha Atioğlu, Halis Karataş and Zülbiye Kökbudak The crystal structure of 5-benzoyl-1-[(E)-(4-

fluorobenzylidene)amino]-4-phenylpyrimidin-2(1H)-one, $\mathrm{C}_{24} \mathrm{H}_{16} \mathrm{FN}_{3} \mathrm{O}_{2}-1039$

Orbett T. Alexander and Johan Venter Crystal structure of monocarbonyl( $\mathrm{N}$-nitroso- $\mathrm{N}$-oxidophenylamine- $\kappa^{2} O, O^{\prime}$ )(tricyclohexylphosphine- $\kappa P$ ) rhodium(I), $\mathrm{C}_{25} \mathrm{H}_{39} \mathrm{~N}_{2} \mathrm{O}_{3}$ PRh -1043

Xi-Ting Xu, Tong-Min Dong, Wei Song and Kai-Long Zhong Crystal structure of poly[bis $\left[\mu_{3}-1,3,5\right.$-tris[(1H-imidazol-1yl)methyl]benzene- $\left.\kappa^{3} N: N^{\prime}: N^{\prime \prime}\right]$ nickel(II)] hexafluorosilicate, $\mathrm{C}_{36} \mathrm{H}_{36} \mathrm{~N}_{12} \mathrm{NiSiF}_{6}-1047$
Jun-Jie Li, Fang-Yun Dong, Qi-Wen Zou, Yin Feng, Yu-Tao Yang, Kai-Xuan Su, Yong-Fu Huang, Xu-Hong Jiang and Xing-An Cheng

The crystal structure of 13-(pyrazole-1-yl-4-carbonitrile)matrine, $\mathrm{C}_{19} \mathrm{H}_{25} \mathrm{~N}_{5} \mathrm{O}-1051$

Xia Zhou, Zhong-Hao Sun, Guang-Zhi Shan and Qing-Guo Meng

Crystal structure of 3,5-bis((E)-4-methoxy-2(trifluoromethyl)benzylidene)-1-methylpiperidin-4-one, $\mathrm{C}_{24} \mathrm{H}_{21} \mathrm{~F}_{6} \mathrm{NO}_{3}-1055$

Nana Xin, Jun Zheng and Qingpeng He The crystal structure of $N, N^{\prime}$-(Disulfanediyldi-2,1phenylene)di(6'-methylpyridine)-2-carboxamide, $\mathrm{C}_{26} \mathrm{H}_{22} \mathrm{~N}_{4} \mathrm{O}_{2} \mathrm{~S}_{2}-1059$

Qing-Guo Meng, Sheng-Nan Zhao, Zhong-Hao Sun, Guang-Zhi Shan and Qing-Guo Meng Crystal structure of (E)-7-fluoro-2-(4-methoxy-2(trifluoromethyl)benzylidene)-3,4-dihydronaphthalen1(2H)-one, $\mathrm{C}_{19} \mathrm{H}_{14} \mathrm{~F}_{4} \mathrm{O}_{2}-1063$

Hong-Yu Song, Shan Liu, Bo-Nan Zhang, Qi-Di Zhong and Ya-Juan Qi

Crystal structure of ethyl 1-(4-fluorophenyl)-4-phenyl-1Hpyrrole-3-carboxylate, $\mathrm{C}_{19} \mathrm{H}_{16} \mathrm{FNO}_{2}-1065$

Orbett T. Alexander, Roger Alberto and Andreas Roodt The crystal structure of cis-diaqua-bis ( $N$-butyl- $N$-(pyridin2-yl)pyridin-2-amine- $\left.\kappa^{2} N, N^{\prime}\right)$ cobalt(II)] dichloride trihydrate, $\mathrm{C}_{28} \mathrm{H}_{44} \mathrm{Cl}_{2} \mathrm{~N}_{6} \mathrm{O}_{5} \mathrm{Co}-1069$

Lei Wang, Qing-Guo Meng, Nan Jiang, Lin Wei and Chun-Hua Wang

Crystal structure of (E)-7-methoxy-2-((6-methoxypyridin3-yl)methylene)-3,4-dihydronaphthalen-1(2H)-one,

$\mathrm{C}_{18} \mathrm{H}_{17} \mathrm{NO}_{3}-1073$

Chen Wang, Lei Wang, Qing-Guo Meng, Zhi-Xin Huang, Nuan-Nuan Ma and Chun-Hua Wang Crystal structure of (E)-2-((3-fluoropyridin-4-yl) methylene)-7-methoxy-3,4-dihydronaphthalen-1(2H)one, $\mathrm{C}_{17} \mathrm{H}_{14} \mathrm{FNO}_{2}-1075$

Lubabalo Ndima, Eric C. Hosten and Richard Betz The crystal structure of 6-bromohexanoic acid, $\mathrm{C}_{6} \mathrm{H}_{11} \mathrm{BrO}_{2}-1079$ 
Pholani Manana, Eric C. Hosten and Richard Betz The crystal structure of 4-chloro-thiophenol, $\mathrm{C}_{6} \mathrm{H}_{5} \mathrm{CIS}-1083$

Pholani Manana, Eric C. Hosten and Richard Betz The crystal structure of 4-bromobenzyl chloride, $\mathrm{C}_{7} \mathrm{H}_{6} \mathrm{BrCl} \longrightarrow 1087$

Pholani Manana, Eric C. Hosten and Richard Betz The crystal structure of di-tert-butyl dicarbonate, $\mathrm{C}_{10} \mathrm{H}_{18} \mathrm{O}_{5}-1091$

Long-Sheng Zou, Zhong-Yan Li and Lin Yuan The crystal structure of (2-(4-chlorophenyl)-5-methyl-1,3dioxan-5-yl)methanol, $\mathrm{C}_{12} \mathrm{H}_{15} \mathrm{ClO}_{3}-1093$

Itumeleng B. Setshedi and Mark G. Smith The crystal structure of the co-crystal: 2-hydroxybenzoic acid - $\boldsymbol{N}^{\prime}$-(butan-2-ylidene)pyridine-4-carbohydrazide, $\mathrm{C}_{10} \mathrm{H}_{13} \mathrm{~N}_{3} \mathrm{O} \cdot \mathrm{C}_{7} \mathrm{H}_{6} \mathrm{O}_{3}-1097$

Jia-Jia Sun, Xiao-Fan Zhang, Qing-Guo Meng, Hong-Juan Li and Chun-Hua Wang

Crystal structure and anti-inflammatory activity of (E)-7fluoro-2-((5-methoxypyridin-3-yl)methylene)-3,4dihydronaphthalen-1(2H)-one, $\mathrm{C}_{17} \mathrm{H}_{14} \mathrm{FNO}_{2}-1101$

Xiang-Yi Su, Xiao-Fan Zhang, Qing-Guo Meng and Hong-Juan Li Crystal structure of (E)-7-fluoro-2-((6-methoxypyridin-3yl)methylene)-3,4-dihydronaphthalen-1(2H)-one, $\mathrm{C}_{17} \mathrm{H}_{14} \mathrm{FNO}_{2}-1105$

Yao-Hong Zhou, Xu-Liang Nie, Wan-Ming Xiong, Da-Yong Peng and Xiao-Hua Zhang

Crystal structure of 1,1'-(butane-1,4-diyl)bis(3-propyl-1Himidazol-3-ium) bis(hexafluoridophosphate),

$\mathrm{C}_{32} \mathrm{H}_{56} \mathrm{~F}_{24} \mathrm{~N}_{8} \mathrm{P}_{4}-1109$
Fei Yang, Zongwei Wang, Pengju Liu, Libing Guo and Dong Xian

The crystal structure of dichlorido-bis(3-methyl-3imidazolium-1-ylpropionato- $\mathrm{K}^{2}$ )-cadmium(II),

$\mathrm{C}_{14} \mathrm{H}_{20} \mathrm{CdCl}_{2} \mathrm{~N}_{4} \mathrm{O}_{4}-1111$

Yi-Da Li, Zhi-Qiang Song, Zhi-Wei Yue, Qi-Di Zhong and Ya-Juan Qi

Crystal structure of 1-(2-cyanobenzyl)-3-cyano-4-phenyl4-(2-cyanobenzyl)-1,4-dihydropyridine monohydrate, $\mathrm{C}_{56} \mathrm{H}_{42} \mathrm{~N}_{8} \mathrm{O}-1113$

Wenqi Song, Miaoxiu Yang, Liwei Qian and Zongcheng Miao

The crystal structure of 3-(carboxymethyl)-1-ethenyl-1Himidazol-3-ium chloride, $\mathrm{C}_{7} \mathrm{H}_{9} \mathrm{~N}_{2} \mathrm{O}_{2} \mathrm{Cl} \longrightarrow 1115$

Jonathan O. Bauer

The crystal structure of

adamantylmethoxydiphenylsilane, $\mathrm{C}_{23} \mathrm{H}_{28} \mathrm{OSi}-1117$

Lucky Dey, Saswata Rabi, Zinnat A. Begum, Tsugiko Takase, Ismail M. M. Rahman, Edward R. T. Tiekink and Tapashi Ghosh Roy

Redetermination of the crystal structure of (2E,4Z,13E,15Z)-3,5,14,16-tetramethyl-2,6,13,17tetraazatricyclo[16.4.0.0 $\left.0^{7,12}\right]$ docosa1(22),2,4,7,9,11,13,15,18,20-decaene, $\mathrm{C}_{22} \mathrm{H}_{24} \mathrm{~N}_{4}-1121$

Lun-Hai Liang, Feng-Lan Zhao and Qing-Guo Meng Crystal structure of (E)-7-hydroxy-2-((6-methoxypyridin2-yl)methylene)-3,4-dihydronaphthalen-1(2H)-one, $\mathrm{C}_{17} \mathrm{H}_{15} \mathrm{NO}_{3}-1125$

Jie Liu, Peng Jiang, Xiao-Ming Yang and Jiu-Fu Lu Crystal structure of catena-poly[diaqua-bis $\left(\mu_{2}-1,3-\mathrm{di}(1 H-\right.$ imidazol-1-yl)propane- $\left.\kappa^{2} N: N^{\prime}\right)$ cobalt(II)] dinitrate, $\mathrm{C}_{18} \mathrm{H}_{28} \mathrm{~N}_{10} \mathrm{O}_{8} \mathrm{Co}-1129$ 